\title{
Robotic surgery: A promising paradigm shift for selected candidates with gynecologic diseases
}

\author{
Jingjing Liu ${ }^{1}$, Xingsheng Yang ${ }^{2}$ \\ ${ }^{1}$ Department of Obstetrics and Gynecology, Yantai Affiliated Hospital of Binzhou Medical University, Yantai; ${ }^{2}$ Department of Obstetrics and \\ Gynecology, Qilu Hospital of Shandong University, Jinan, China
}

In recent years, with the concepts of minimally invasive surgeries (MIS), the treatment of diseases has gradually evolved from traditional open surgery to endoscopic minimally invasive surgery. Since the first generation of Da Vinci robots was developed in 1996, by 2019, the cumulative number of Da Vinci robots worldwide has reached 5,582. Among them, 596 units were added in 2019, a year-onyear increase of $11.95 \%$ [1]. On average, every 26 seconds in the world, a doctor uses Da Vinci for surgery.

Recently, an original article in this issue studied over 12,000 surgical cases from the 14 hospitals about the utilization rate of robot platforms during the past 13 years in South Korea [2]. The annual data showed the rate and volume for both single-site and multiport robotic surgeries increased steadily in benign and malignant tumors. In gynecological surgery, including gynecological malignant tumors, the scope of approved surgery is getting wider and wider, and the amount of surgery is increasing year by year. It greatly expands the skill boundaries of surgeons, significantly reduces the work intensity of surgeons, completes surgical actions far exceeding human accuracy, greatly in- creases the scope of surgical applications, and increases the success rate of surgery.

Despite the early-stage cervical cancer in Laparoscopic Approach to Carcinoma of the Cervix trial which is a challenge for MIS due to the lower disease-free survival and overall survival rates compared to open radical hysterectomy surgeries published in 2018. This disadvantage might be minimized by reducing the tumor traction and careful colpotomy with the skillful surgeons.

Although the cost-effectiveness of robot-assisted surgery is still debatable, there are numerous robotic device companies rushing into the market with a lower price, e.g., REVO-1 system from Mere company is one of these suppliers with a lower price compared with the Da Vinci systems. It is believed that robotic surgery system is a boon to surgeons and both surgeons and patients' lives will benefit a lot from it.

\section{References}

1. Intuitive Surgical. Annual Report 2019 [Internet]. Sunnyvale (CA): Intuitive Surgical; c2020 [cited 2020 MON DAY]. Avail-

- Received: September 21, 2020 • Accepted: September 22, 2020

- Correspondening author: Xingsheng Yang

Department of Obstetrics and Gynecology, Qilu Hospital of Shandong University, No.107 Wenhua Xi Road, Jinan, Shandong 250012, China E-mail:xingshengyang@sdu.edu.cn

This is an Open Access article distributed under the terms of the Creative Commons Attribution Non-Commercial License (http://creativecommons.org/ licenses/by-nc/4.0) which permits unrestricted non-commercial use, distribution, and reproduction in any medium, provided the original work is properly cited. 
Gynecologic Robotic Surgery | Vol 1, No. 2, September 2020

able from: https://www.annualreports.com/HostedData/AnnualReports/PDF/NASDAQ_ISRG_2019.pdf.

2. Lee S, Kim MR, Seong SJ, Paek J, Lee YS, Nam EJ, et al. Trends in robotic surgery in Korean gynecology. Gyne Robot Surg 2020;1:50-56. 